\title{
Code S: Redesigning Hospital-Wide Peer Review Processes to Identify System Errors
}

Huy D. Au ${ }^{1}$, Daniel I. Kim ${ }^{2}$, Roger C. Garrison ${ }^{1}$, Minho Yu ${ }^{1}$, Gary Thompson ${ }^{1}$, Ramiz Fargo ${ }^{1}$, Brandon Nathaniel ${ }^{1}$, Morteza Chitsazan ${ }^{1}$, Lakshmi K. Puvvula ${ }^{1}$, Ali Motabar ${ }^{1}$, Lawrence K. Loo ${ }^{3}$

1. Internal Medicine, Riverside University Health System Medical Center, Moreno Valley, USA 2. Internal Medicine, University of California Riverside School of Medicine, Moreno Valley, USA 3. Internal Medicine, Loma Linda University School of Medicine, Loma Linda, USA

Corresponding author: Huy D. Au, h.au@ruhealth.org

\begin{abstract}
Hospital medical errors that result in patient harm and death are largely identified as system failures. Most hospitals lack the tools to effectively identify most system errors. Traditional methods used in many hospitals, such as incident reporting (IR), departmental morbidity and mortality conferences, and root cause analysis committees, are often flawed by under reporting. We introduced the Code S designation into our hospital's ongoing physician peer review process as an additional and innovative way to identify system errors that contributed to adverse clinical outcomes. The authors conducted a retrospective review of all peer review cases from January 2008 to December 2011 and determined the quantity and type of system errors that occurred. System errors were categorized based on a modified $5 \mathrm{M}$ model which was adapted to reflect system errors encountered in healthcare. The Code S designation discovered 204 system errors that otherwise may not have previously been identified. The addition of the Code S designation to the peer review process can be readily adopted by other healthcare organizations as another tool to help identify, quantify and categorize system errors, and promote hospital-wide process improvements to decrease errors and improve patient safety.
\end{abstract}

Categories: Quality Improvement

Keywords: peer review, system errors, quality improvement, hospital processes

\section{Introduction}

Medical errors commonly occur in the hospital setting and result in patient harm and even death [1-3]. A 2016 study from John Hopkins suggested that medical errors were the third leading cause of death in the US, which translated to an estimated 250,000 or more deaths per year [4]. A large majority of medical errors resulting in patient harm are related to system failures, and not from individual errors or negligence [5-8].

Received 05/16/2020 Review began 05/21/2020 Review ended 05/29/2020 Published 06/05/2020

\section{() Copyright 2020}

Au et al. This is an open access article distributed under the terms of the Creative Commons Attribution License CC-BY 4.0., which permits unrestricted use, distribution, and reproduction in any medium, provided the original author and source are credited.
Originally developed by the medical profession to review the qualifications and practice patterns of medical staff physicians $[9,10]$, physician peer review can be used to identify system errors that may contribute to adverse clinical outcomes $[11,12]$. Unfortunately, the review process to characterize system errors is limited, due to vastly divergent approaches used to conduct physician peer reviews within various healthcare organizations $[9,13]$.

Given the significant negative impact of system-related medical errors on patient safety, many healthcare organizations have established medical error reduction as a priority. This has strengthened the case for developing a systematic process to recognize and subsequently prevent system-related medical errors. The Joint Commission (TJC) maintains that meaningful improvements in patient safety are dependent on each organization's ability to identify errors and analyze their contributing factors to prevent similar errors from recurring [8].

In 2008, Riverside University Health System - Medical Center (RUHS-MC) modified its hospital-wide physician peer review process to better capture system errors, an approach that RUHS-MC still uses today. All physician peer reviewers were required to report system errors that may have contributed to an unexpected or adverse event [14]. Code S (for "Systems") designation was given to physician peer review cases that identified a contributing system error(s). The purpose of this retrospective chart review study was to (1) demonstrate the feasibility of using the Code $S$ designation in a hospital-wide physician peer review process to identify and track system errors, (2) determine the proportion of reviewed cases that were classified as a Code S designation, and (3) characterize and identify the most common types of system errors identified using our modified 5M model.

\section{Materials And Methods}

\section{Hospital description}

RUHS-MC is a 439-bed academic safety net hospital and level II trauma center in Moreno Valley, California. 
The patient population is a low income, predominantly Hispanic population. RUHS-MC is an important site for graduate medical education with residency programs in the specialties of internal medicine, family medicine, anesthesiology, orthopedic surgery, general surgery, neurosurgery, pediatrics, emergency medicine and obstetrics/gynecology. RUHS-MC is also an important site for undergraduate medical education with medical students rotating from three medical schools in the region, University of California, Riverside (UCR), Loma Linda University and Western University of Health Sciences. The medical staff consists of approximately 450 attending physicians.

\section{Peer review process}

The institutional peer review process spans all departments within the hospital and is performed as part of the Ongoing Professional Practices Evaluation (OPPE) system as recommended by TJC [15]. Cases selected for peer review were based on established RUHS policy and included patient deaths, unexpected adverse outcomes, incident reports, quality measure fallouts, routine peer reviews for medical staff re-credentialing, and selected reviews of practitioner performance as determined by the individual departments as recommended by TJC for hospital peer review processes [16]. Physicians providing peer review were medical staff appointed by their respective departments and excluded if they were involved in the case. The peer reviewers completed a standardized peer review form to assign quality of care levels per hospital protocol (Figure 1). In cases where a significant system deficit contributed to a medical error, the peer reviewer would assign a preliminary Code S and document the details of the potential system error(s) that may have contributed to an adverse outcome(s).

RIVERSIDE UNIVERSITY HEALTH SYSTEM

PEER REVIEW AND DOCUMENTATION REVIEW FORM

\begin{tabular}{|c|c|c|c|}
\hline \multicolumn{2}{|c|}{ Department / Service: } & \multicolumn{2}{|c|}{ Date of Initial Review / Referral: } \\
\hline \multicolumn{2}{|c|}{ Medical Record \#: } & & Discharge: \\
\hline \multicolumn{2}{|c|}{ Patient Age: } & \multicolumn{2}{|l|}{ Patient Sex: } \\
\hline \multicolumn{4}{|c|}{ Attending Physician \#: } \\
\hline \multicolumn{4}{|c|}{ Admitting Diagnosis: } \\
\hline \multicolumn{4}{|c|}{ Reason for Review: } \\
\hline \multicolumn{2}{|c|}{ Issue Identification: Check all that apply } & \multicolumn{2}{|l|}{ Due Date: } \\
\hline A & No issues with physician quality of care & \multirow{2}{*}{\multicolumn{2}{|c|}{$\begin{array}{l}\text { Check Appropriate Peer Review Level for } \\
\text { Quality of Care }\end{array}$}} \\
\hline B & No issues with documentation & & \\
\hline \multirow{2}{*}{\multicolumn{2}{|c|}{$\begin{array}{l}\text { If A\&B are marked, do not check C-O } \\
\text { and select Level I }\end{array}$}} & Levell & Meets Medical Staff Standard of Care \\
\hline & & Level II & $\begin{array}{l}\text { Meets Standard of Care. Opportunity for } \\
\text { Improvement Exists. See Below }\end{array}$ \\
\hline \multicolumn{2}{|c|}{$\begin{array}{l}\text { Physician Care Issues: Check all that apply. } \\
\text { If C-O are marked, select Level II, III, or IV }\end{array}$} & Level III & \multirow{3}{*}{\begin{tabular}{|l|} 
Deviation to Standard of Care. See Below \\
Episode of Care Unacceptable to Medical \\
Staff. See Below
\end{tabular}} \\
\hline \multicolumn{2}{|c|}{ Patient Care } & \multirow{2}{*}{ Level IV } & \\
\hline $\mathrm{C}$ & Diagnosis & & \\
\hline $\mathrm{D}$ & Clinical Judgment / Decision-making & \multicolumn{2}{|c|}{\begin{tabular}{|l|l|} 
& Staff. See Below \\
\end{tabular}} \\
\hline $\mathrm{E}$ & \multirow{2}{*}{\begin{tabular}{|l|} 
Technique / Skills \\
Diagnostic / Treatment Planning
\end{tabular}} & \multirow{2}{*}{\multicolumn{2}{|c|}{$\begin{array}{c}\text { Check "Code S and/or R" if System or Resident issue } \\
\text { involved in quality of care. Must also assign a } \\
\text { Peer Review Level }\end{array}$}} \\
\hline $\mathrm{F}$ & & & \\
\hline G & Follow-up / Follow-through & \multirow[t]{2}{*}{$\underline{\text { Code } \mathbf{S}}$} & \multirow{2}{*}{$\begin{array}{l}\text { Opportunity for Improvement Exists - Not } \\
\text { Related to Provider's Performance: } \\
\text { System Problem. See Below }\end{array}$} \\
\hline \multicolumn{2}{|r|}{ Medical Knowledge } & & \\
\hline $\mathrm{H}$ & Clinical Knowledge & \multirow{3}{*}{$\begin{array}{l}\text { Code R } \\
\text { or AHP }\end{array}$} & \multirow{3}{*}{$\begin{array}{l}\text { Opportunity for Improvement Exists - } \\
\text { Related to resident performance. See } \\
\text { Below }\end{array}$} \\
\hline \multicolumn{2}{|r|}{ Professionalism } & & \\
\hline I & Supervision (Resident Physician or AHP) & & \\
\hline & erpersonal and Communication Skills & & de S or R" Explanation of Issues \\
\hline $\mathrm{J}$ & Communication / Responsiveness & & \\
\hline & Systems-Based Practice & Resident/AF & Provider \#: \\
\hline K & Policy Compliance & & \\
\hline $\mathrm{L}$ & Other & & \\
\hline Physicia & Documentation: Check all that apply & & \\
\hline Int & erpersonal and Communication Skills & & \\
\hline$M$ & Documentation reflects patient condition & & \\
\hline $\mathrm{N}$ & Documentation unreadable & & \\
\hline 0 & Documentation incomplete & & \\
\hline
\end{tabular}

1. List the opportunities for improvement identified for Peer Review Levels II, III, IV, S or R:

2. List the actions taken for Peer Review Levels II, III, IV, S or R:

3. Pending next department meeting $\square$

Signature of Reviewer: Date:

MEC Approved: 06//14/12

PRIVILEGED AND CONFIDENTIAL - NOT FOR RELEASE Peer Review Documents are Protected by Evidence Code 1157

FIGURE 1: Peer review and documentation review form 
All peer reviews were conducted and adjudicated at the individual department level. After the initial departmental reviews, the peer reviews were forwarded to the Quality Management Department (QMD) and then reviewed by the Professional Practices Evaluation Committee (PPEC), which was comprised of medical staff from multiple hospital departments, QMD, nursing staff and administration. The PPEC was responsible for determining a final quality level including the Code $\mathrm{S}$ designation for each peer-reviewed case. If the PPEC disagreed with the initial departmental review, the case was sent back to the department for reassessment. The PPEC also sent inquiries to the appropriate departments to address the system issues identified by the Code S designation. The PPEC closed the review of the Code $\mathrm{S}$ once the issues were addressed and corrected (Figure 2). Once the peer reviews were finalized, the information was entered into the QMD database. As the QMD was responsible for performing the root cause analysis, not the initial peer reviewer, very little training was required at the individual provider level.

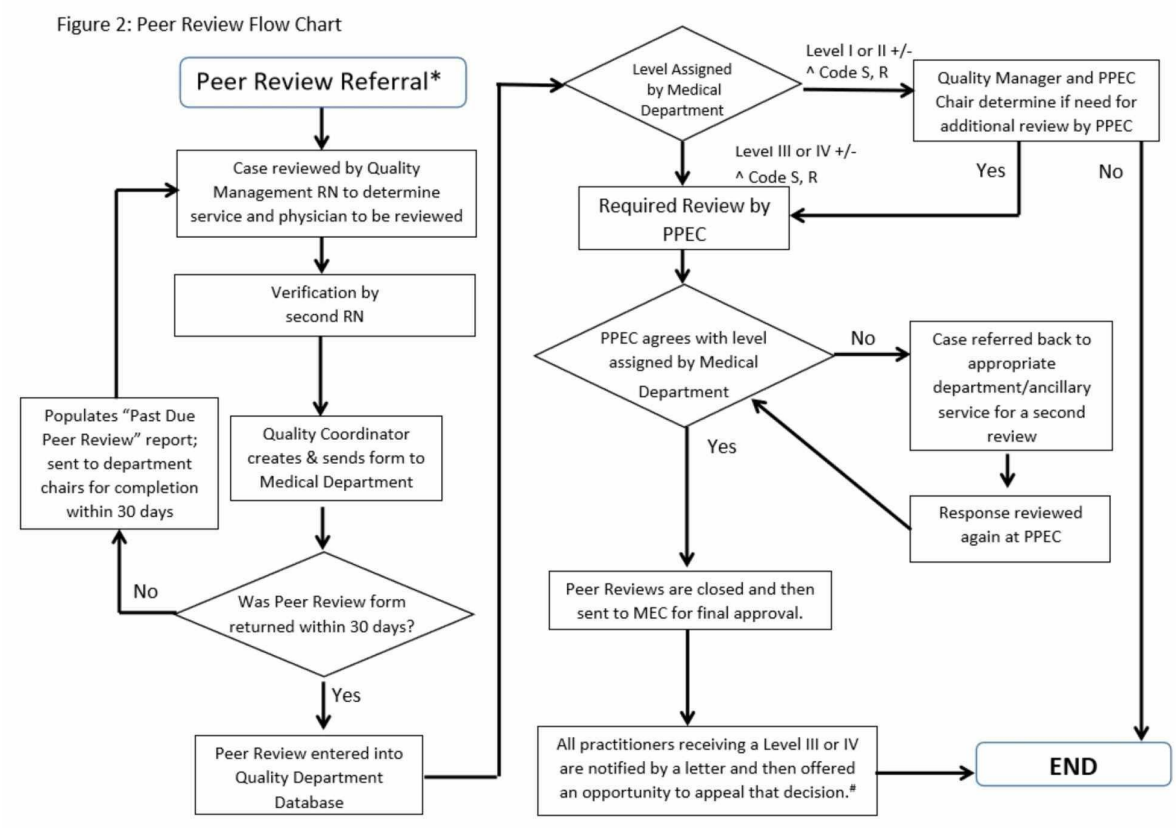

\section{FIGURE 2: Peer review flow chart}

PPEC - Professional Practices Evaluation Committee; MEC - Medical Executive Committee

\section{Study protocol}

To evaluate the feasibility and outcome of this peer review process, we conducted a retrospective crosssectional quality improvement project utilizing peer reviews conducted from January 2008 to December 2011 by the QMD at RUHS-MC. In January 2008, we began assigning Code $\mathrm{S}$ as well as Code R (resident) designations to our peer reviews, a process we still utilize today. We selected this date range to coincide with our prior Code R study focusing on potential errors involving resident trainees [14]. The study was reviewed by the RUHS Institutional Review Board and was designated a quality improvement project, which did not require formal review.

Members of the Code S study team evaluated all peer reviews that were assigned a Code $\mathrm{S}$ designation between 2008 and 2011 from the QMD database to determine the characteristics of the system errors. Data collected included peer review level assigned, area of deficiency, originating department and whether there was a Code S designation. Two reviewers independently evaluated and assigned the category of deficiency based on a modified $5 \mathrm{M}$ framework [7,17]. The reviewers were instructed to review the underlying cause of the deficiency and assign it to one or more of the modified 5M categories. In the cases where the two reviewers assigned different categories to the Code S error, they would discuss the review between themselves and attempt to reach a consensus. In two cases, where consensus was not met between the two reviewers, a third reviewer adjudicated the case to determine the final category assigned.

Our study used the $5 \mathrm{M}$ model as a framework to categorize the physician peer reviews with a Code $\mathrm{S}$ designation. The $5 \mathrm{M}$ model is a well-known risk management tool, primarily used in the manufacturing and aviation industries, to analyze adverse events, and has been successfully adapted to the field of medicine $[7,17]$. In this study, we modified the traditional $5 \mathrm{M}$ model to better reflect the system errors commonly 


\section{Cureus}

encountered in hospitals.

Man: human factors contributing to the event (e.g. communication, staffing, training)

Machine: tools and equipment used in patient care

Medium: environmental aspects that occur locally within the hospital that contributed to the event

Materials: medications and supplies related to patient care

Management: managerial aspects such as regulations and policies

External Matters: issues outside of the control of the hospital

Data analyses used descriptive statistics including chi-square, Fisher exact, and t-tests. Due to multiple hypotheses testing, the statistical significance was set a priori at $\mathrm{p}<0.01$.

\section{Results}

We evaluated 8,165 peer-reviewed cases between January 2008 and December 2011. Incomplete peer reviews were removed (four Code S and 353 non-Code S). The final data sets included 204 Code S cases and were compared to 8,106 non-Code $\mathrm{S}$ cases.

Table 1 compares the peer-reviewed designations for each of the four levels. For those cases designated Code S, only $23.1 \%$ were assigned a Level I (Standard of Care), compared to $88.5 \%$ for those designated nonCode S cases. For those cases designated Code S, over 15\% were assigned a Level III (Deviation from Standard of Care) or Level IV (Unacceptable Care), compared to less than 1\% of those designated non-Code S cases, $\mathrm{p}<0.0001$. The Code $\mathrm{S}$ designation was associated with an odds ratio of 23.5 greater likelihood that the two lowest levels of peer-reviewed quality of care (Levels III or IV - "Deviation from Standard of Care" or "Unacceptable Care") would be identified compared to non-Code S cases. There were no significant differences in the patients' age or gender among the different departments.

\begin{tabular}{|c|c|c|c|c|c|c|c|c|c|}
\hline & \multicolumn{4}{|c|}{ Code S } & \multicolumn{4}{|c|}{ Non-Code S } & P Value \\
\hline Number of Cases & \multicolumn{4}{|l|}{204} & \multicolumn{4}{|l|}{7812} & \\
\hline \multicolumn{10}{|l|}{ Age } \\
\hline Average + S.D. & \multicolumn{4}{|c|}{$38.9+22.6$} & \multicolumn{4}{|c|}{$40.2+23.5$} & \multirow{2}{*}{$p=0.427$} \\
\hline Range & \multicolumn{4}{|c|}{$0-93$} & \multicolumn{4}{|c|}{$0-101$} & \\
\hline \multicolumn{10}{|l|}{ Sex (M/F) } \\
\hline Number & \multicolumn{4}{|c|}{$93 / 111$} & \multicolumn{4}{|c|}{$3469 / 3911^{\#}$} & \multirow{3}{*}{$p=0.69$} \\
\hline Percentage & \multicolumn{4}{|c|}{$45.6 \%$ / 54.4\% } & \multicolumn{4}{|c|}{$52.3 \% / 54.7 \%$} & \\
\hline \multicolumn{9}{|l|}{ Peer Review } & \\
\hline Levels* & 1 & II & III & IV & 1 & II & III & IV & \\
\hline Number & 47 & 123 & 28 & 6 & 6916 & 830 & 64 & 2 & \multirow{2}{*}{$p<0.001$} \\
\hline Percentage & 23.1 & 60.3 & 13.7 & 2.9 & 88.5 & 10.6 & 0.8 & 0.02 & \\
\hline
\end{tabular}

\section{TABLE 1: Comparison of Code $\mathbf{S}$ and non-Code $\mathbf{S}$ peer review charts and quality of care}

* Peer Review Levels = I (meets standard of care), 2 (opportunity for improvement), 3 (deviation to standard of care), and 4 (episode of care unacceptable to medical staff).

\# Numbers may not equal total non-Code $\mathrm{S}$ cases due to missing values (but the number of missing values is $<5 \%$ of total).

Figure 3 categorizes the causes of the Code S error based on our modified version of the $5 \mathrm{M}$ model. Among the six causes of system errors, the most common cause identified was "Man" (non-physician human error), which was identified in $58.4 \%$ of the cases, while the second most common cause identified was "Management", accounting for $20.2 \%$ of the cases. Table 2 provides a list of examples of the different types 


\section{Cureus}

of Code S errors.

Figure 3: Code S Categories based on the " $5 \mathrm{M}$ " Model

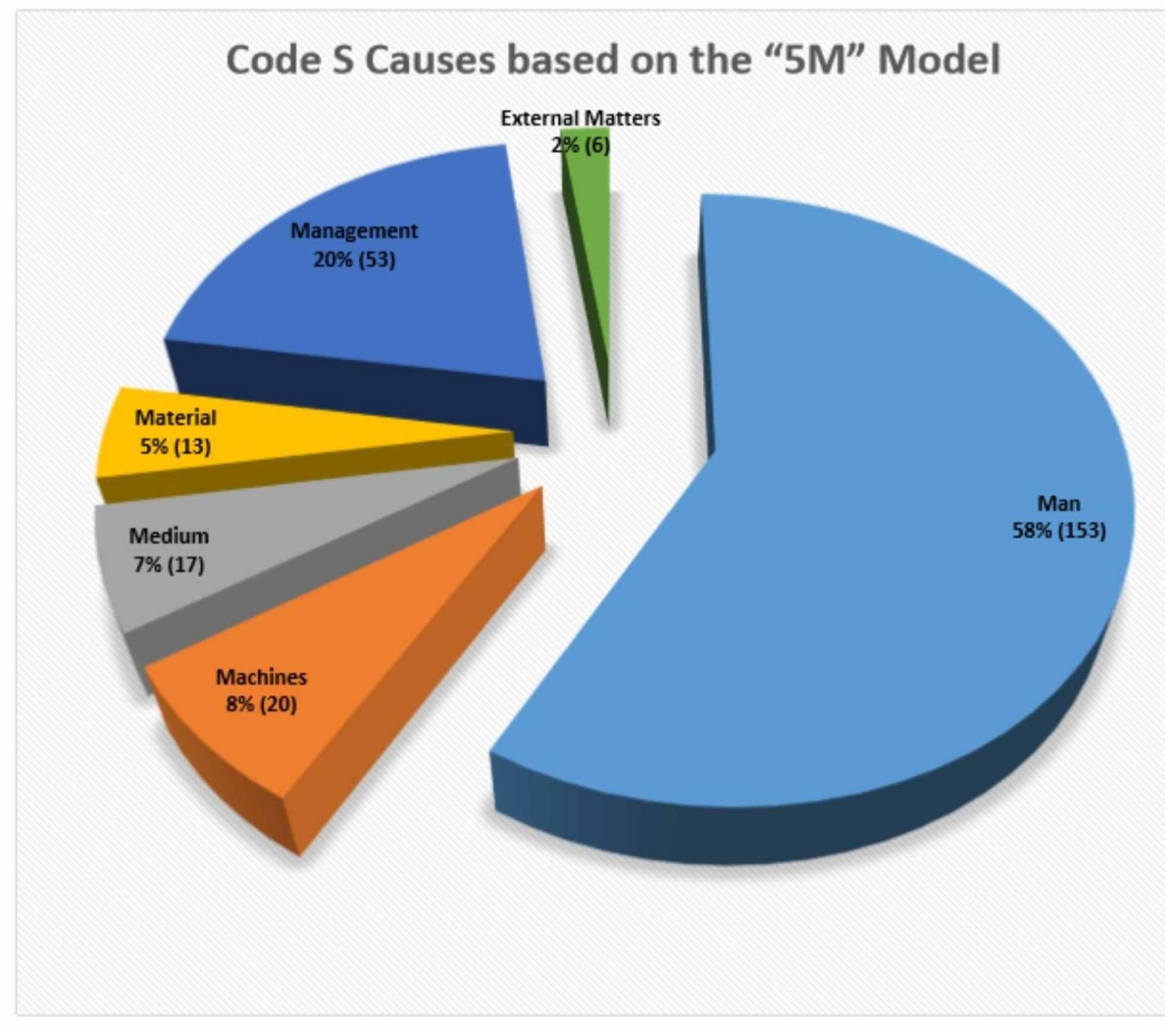

Total Number $=262$. Totals exceed number of cases since multiple causes could contribute to one case.

FIGURE 3: Code $S$ categories based on the " $5 \mathrm{M}$ " model 


\section{Cureus}

Table 2: Examples of each type of Code $\mathrm{S}$

Man - Communication, staffing, level of training, fatigue

Miscommunication between staff members during handoffs

Surgical subspecialty is only staffed with 1 resident and 1 attending for both clinic and emergent procedures. Emergency surgery had to be delayed until the Attending could be freed up from clinic.

Machines - Tools and equipment used in patient care

Ventilator malfunction results in the patient going apneic and subsequently going into a cardiac arrest.

Consulting service orders a send-out test, which is not reported in the hospital EMR resulting in delays in making the diagnosis and initiating the treatment.

Management

Patient coded in the MICU but Anesthesia team did not respond assuming the MICU team would handle the airway. Both MICU Attendings were occupied in procedures and could not respond to the code resulting in a Medicine resident being the most senior person to manage the advance airway insertion.

Materials - Medications and supplies

The OR ran out of Urologic irrigate during a procedure resulting in the OR staff trying to make a similar solution during the case. Normal saline was used instead of sterile water resulting in electrolyte abnormalities postoperatively.

Medium - Uncontrollable events

Extremely high patient volumes during flu season resulting in Emergency Department delays for the average patient.

Drunk driver crashes into the power lines for the hospital resulting in the hospital being dependent on the emergency generators. Available computers become disabled resulting in delays with routine care.

External Matters - issues outside the control of the hospital

Paramedics did not appropriately start CPR on a patient prior to the patient's arrival to the hospital.

TABLE 2: Examples of each type of Code $S$

\section{Discussion}

Approximately 85\% of medical errors that occur in hospitals are related to system errors [5-8]. To identify these system errors, hospitals have traditionally relied on limited and confidential forums, such as departmental morbidity and mortality conferences and root cause analysis committees [4].

More recently, hospitals have also implemented the use of incident reporting (IR) systems to help identify patient safety risks related to system errors. Unfortunately, despite these efforts, IR systems capture only a small fraction of total patient safety events, with as few as $7 \%$ of all events being reported through IR systems [18].

Chassin et al stated "newer and more effective strategies and tools are needed to identify system errors and to address the complex quality and patient safety challenges facing hospitals today" [19]. Hospital-wide physician peer review processes already exist in hospitals to track provider performances; however, they are not routinely used to identify hospital system errors that contribute significantly to adverse patient outcomes or create potential risks for future events.

Another innovation is our modification of the 5M model, commonly used in other industries, which allows it to be more relevant for identifying and categorizing medical errors. Our study found the most common classification of system error was "Man", which represented 58.4\% of the total Code S categories. This finding is supported by existing literature indicating that ineffective communication among health care professionals is the leading cause of medical errors and patient harm and emphasizes the importance of creating strategies to enhance teamwork and communication in the hospital setting [20].

Our results demonstrate the feasibility of integrating a Code S designation into the hospital-wide physician peer review process to provide an additional tool to identify system errors. This innovation goes beyond the traditional methods available to hospitals to identify and prevent system errors and represents a novel way to identify system errors that may otherwise go unrecognized. We reviewed the first three years of the data 
since the Code S designation was instituted starting from 2008 to 2011 . This program has been very successful and continues to lead to systems changes to date. While this method is not designed to identify all hospital system errors, it is a tool that can be easily integrated into an existing peer-review process to help identify system errors that other hospital processes may not identify.

Our study also demonstrates a potential gap in existing physician peer review processes. An adverse event could be incorrectly attributed to substandard physician care; when, in fact, a system-related medical error may be involved. For instance, a physician may have ordered a medication for the wrong patient. Upon further investigation, it was discovered that the electronic medical record (EMR) system was down, and the physician ordered the medication on paper without the correct patient identifiers. In this case, the physician peer review would indicate a Level IV with a Code S designation, since the system certainly was a contributing factor to the medical error. In our opinion, the use of the Code $\mathrm{S}$ designation when performing peer reviews can serve to unmask system errors that were previously unrecognized.

This interdepartmental multidisciplinary system has the added benefit of allowing for a birds-eye view of errors within the hospital instead of having system errors and possible solutions siloed within individual departments. As the QMD and PPEC oversee information from all individual departments, they can more readily review and identify interdepartmental issues and trends. One such example is when three cases of shoulder dystocia occurred and physical therapy was not consulted by the Pediatrics department. A root cause analysis identified that Labor and Delivery had changed to a different informatics system where the physical therapy evaluation was not incorporated in the documentation. The Labor and Delivery nurses were educated to include discussion of shoulder dystocia as a requirement for Labor and Delivery/post-partum handoff.

In addition, our finding that Level III (Deviation to Standard of Care) or Level IV (Unacceptable Care) designations were 24 times more likely when a Code $\mathrm{S}$ was designated, may suggest that system errors are typically associated with more serious medical errors and worse patient outcomes. In addition, the Code S designation may suggest the presence of underlying system variables that place physicians and patients in higher-risk situations. The magnitude of this association may indicate the importance of identifying systemrelated issues, which if corrected, could result in a significant reduction in adverse events.

One limitation is that this study was done at a single institution and may not be representative of other organizations and their peer review processes. Other limitations include potential reviewer bias, a low number of system errors relative to the total number of peer-reviewed cases, and missing peer review levels in our database.

The Code S classifications may have introduced reviewer bias; however, we attempted to limit this potential bias by having two physicians independently assess each case. In the two cases where a consensus was not met, a third reviewer independently reviewed the case and made a final decision. Also, our protocol included a multidisciplinary peer review process for applying the Code S designations which reduced the potential for single reviewer bias or error.

While the data showed a low percentage of system errors identified from the total number of peer reviews, we believe the percentage would have been higher if routine peer reviews for medical staff re-credentialing, which significantly increased the peer review denominator, were removed and only the incident triggered peer reviews were analyzed. However, the database did not allow for this type of analysis.

Another limitation to this process is the possibility of false negatives. The initial reviewer could have failed to identify a system error that contributed to the peer review. However, we attempted to mitigate this by virtue of a step-wise process. After the initial reviewer evaluated the case, it was re-evaluated by the department, voted on and then sent to QMD and PPEC for final review and approval. This process affords multiple opportunities for a system issue to be identified not only by the initial reviewer, but by other physicians in the department, as well as the multidisciplinary QMD and PPEC members.

While there were missing peer review levels in our database, which were excluded from the analysis, $(1.9 \%$ with Code S designation and $4.3 \%$ without Code $\mathrm{S}$ designation), they represented a small percentage of the overall data set and were not felt to have a significant impact on the results.

\section{Conclusions}

System errors are the most common types of errors within hospitals. Despite this fact, most system errors are not rigorously identified and systematically categorized in most hospital-wide peer review processes. Unfortunately, there is no tool that can identify all hospital system errors. Having more effective methods for identifying system errors is the first step that allows hospitals to more effectively combat and reduce system errors. Identification of system errors by peer review can be a helpful adjunct in identifying safety and quality of care issues in hospitals and facilitate system changes to improve patient care and decrease risk for patient harm. To our knowledge, this is the first tool that integrates the identification and evaluation of system errors into the hospital-wide peer review process. The addition of the Code S designation to the 
peer review process can be readily adopted by other healthcare organizations as an effective tool to help identify, quantify and categorize system errors, and promote hospital-wide process improvements to decrease medical errors and improve patient safety.

\section{Additional Information \\ Disclosures}

Human subjects: All authors have confirmed that this study did not involve human participants or tissue. Animal subjects: All authors have confirmed that this study did not involve animal subjects or tissue. Conflicts of interest: In compliance with the ICMJE uniform disclosure form, all authors declare the following: Payment/services info: All authors have declared that no financial support was received from any organization for the submitted work. Financial relationships: All authors have declared that they have no financial relationships at present or within the previous three years with any organizations that might have an interest in the submitted work. Other relationships: All authors have declared that there are no other relationships or activities that could appear to have influenced the submitted work.

\section{References}

1. Leape LL: Error in medicine. JAMA. 1994, 272:1851-1857. 10.1001/jama.1994.03520230061039

2. Brennan TA, Leape LL, Laird NM, et al.: Incidence of adverse events and negligence in hospitalized patients -results of the Harvard Medical Practice Study I. N Engl J Med. 1991, 324:370-376. 10.1056/NEJM199102073240604

3. Leape LL, Brennan TA, Laird N, et al.: The nature of adverse events in hospitalized patients-results of the Harvard Medical Practice Study II. N Engl J Med. 1991, 324:377-384. 10.1056/NEJM199102073240605

4. Makary, MA, Daniel M: Medical error-the third leading cause of death in the US . BMJ. 2016, 353:i2139. 10.1136/bmj.i2139

5. Al-Lamki L: Peer review of physicians' performance: is it a necessary quality assurance activity? . Sultan Qaboos Univ Med J. 2009, 9:109-112.

6. Reason J: Human error: models and management. BMJ. 2000, 320:768-770. 10.1136/bmj.320.7237.768

7. Wilf-Miron R, Lewenhoff I, Benyamini Z, Aviram A: From aviation to medicine: applying concepts of aviation safety to risk management in ambulatory care. BMJ Qual Saf. 2003, 12:35-39. 10.1136/qhc.12.1.35

8. Sanchez JA, Lobdell KW, Moffatt-Bruce SD, Fann JI: Investigating the causes of adverse events . Ann Thorac Surg. 2017, 103:1693-1699. 10.1016/j.athoracsur.2017.04.001

9. Edwards MT, Benjamin EM: The process of peer review in U.S. hospitals . J Clin Outcomes Manage. 2009, 16:461-467.

10. Simmons TA, Winstead AB: The Future of Peer Review: Position Candor Versus Patient Right . DRI, Chicago, IL; 2009.

11. Guthrie M: Guidelines for disciplinary action from peer review . Physician Exec. 2009, 35:78-79.

12. Parker M: Embracing the new professionalism: self-regulation, mandatory reporting and their discontents . J Law Med. 2011, 18:456-466.

13. Chan LS, Elabiad M, Zheng L, et al.: A medical staff peer review system in a public teaching hospital--an internal quality improvement tool. J Healthc Qual. 2014, 36:37-44. 10.1111/j.1945-1474.2012.00208.x

14. Kim DI, Au H, Fargo R, Garrison RC, Thompson G, Yu M, Loo LK: Code R: redesigning hospital-wide peer review for academic hospitals. Am J Med Qual. 2016, 31:429-433. 10.1177/1062860615583548

15. Ongoing Professional Practice Evaluation (OPPE) - intent. (2016). Accessed: May 1, 2020: https://dev.jointcommission.org/en/standards/standard-faqs/critical-access-hospital/medical-staff$\mathrm{ms} / \mathrm{ka044000000y0jw/.}$

16. Ongoing Professional Practice Evaluation (OPPE) - data collection guidelines and frequency . (2016). Accessed: May 1, 2020: https://www.jointcommission.org/standards_information/jcfaq.aspx.

17. 5-M method. (2016). Accessed: May 1, 2020: https://managementmania.com/en/5-m-method.

18. De Feijter JM, de Grave WS, Muijtjens AM, Scherpbier AJ, Koopmans RP: A comprehensive overview of medical error in hospitals using incident-reporting systems, patient complaints and chart review of inpatient deaths. PLoS One. 2012, 7:31125. 10.1371/journal.pone.0031125

19. Chassin MR: Improving the quality of health care: what's taking so long? . Health Aff. 2013, 32:1761-1765. 10.1377/hlthaff.2013.0809

20. Henriksen K, Battles JB, Keyes MA, Grady ML: Advances in Patient Safety: New Directions and Alternative Approaches. Healthcare Research and Quality, Rockville, MD; 2008. 